\title{
Difference Analogues of Quasi-Linear Elliptic Dirichlet Problems with Mixed Derivatives*
}

\author{
By Robert S. Stepleman
}

\begin{abstract}
In this paper we consider a class of difference approximations to the Dirichlet problem for second-order quasi-linear elliptic operators with mixed derivative terms. The main result is that for this class of discretizations and bounded $g$ (the right-hand side) a solution to the difference equations exists. We also explicitly exhibit a discretization of this type for a class of operators.
\end{abstract}

1. Introduction. In this paper we will be concerned with obtaining existence results for solutions of nonlinear systems of $n$ equations in $n$ unknowns which arise in a natural way from the study of the following problem. Let $\Omega$ be an open, bounded, and simply connected region in the plane, and let $\psi: \partial \Omega \rightarrow R^{1}$. Then, find $u \in C^{2}(\Omega) \cap$ $C(\bar{\Omega})$ such that

$$
\begin{aligned}
L u & =g\left(s, t, u, u_{s}, u_{t}\right), & & (s, t) \in \Omega, \\
u & =\psi, & & (s, t) \in \partial \Omega,
\end{aligned}
$$

where $L$ is a second-order quasi-linear elliptic operator of the form

$$
L u=A u_{s s}+2 B u_{s t}+C u_{t t} .
$$

Here $A, B$, and $C$ depend, in general, on $s, t, u, u_{s}$ and $u_{t}$, but not on the second derivatives.

To treat the problem (1.0), we use arbitrary consistent finite-difference approximations to the various derivatives, which yield nonlinear finite-dimensional operators of "positive type"; this extends methods of discretizing linear elliptic partial-differential equations so as to yield operators of positive type which have been studied by Motzkin and Wasow [7], and Bramble and Hubbard [2], as well as others. By these discretization methods, we obtain nonlinear systems of equations as approximations to (1.0); and then apply fixed point techniques to these systems to show the solutions exist. In doing this, we generalize several results of Bers [1]. We also extend a discretization given by Bramble and Hubbard [2], which gives operators of positive type for linear uniformly elliptic problems, to the largest class of quasi-linear elliptic problems for which it gives operators of positive type. In doing this, we correct several errors in Frank [4], which was an attempt to extend this discretization to all quasi-linear

Received May 1, 1970.

AMS 1969 subject classifications. Primary 6562.

Key words and phrases. Difference analogues, elliptic, mixed derivatives.

* This research was supported in part by the National Aeronautics and Space Administration under Grant NSG 398 to the Computer Science Center of the University of Maryland and by the U. S. Army Research Office Durham under Grant DA-HCO4-(AROD)-67-C-0062 to the Institute for Fluid Dynamics and Applied Mathematics of the University of Maryland. This research was also supported in part by the Office of Naval Research under grant NONR474(14).

Copyright @ 1971, American Mathematical Society 
uniformly elliptic problems. The problem of discretizing quasi-linear elliptic operators to obtain operators of positive type has also been considered by McAllister [5], [6]; our results are in a broad sense generalizations of the results there.

2. Affine Operator-Valued Mappings. The main tool we will use in obtaining our existence theorems for solutions to discretized versions of Dirichlet problems is a result about affine operator-valued mappings. We now proceed to develop some needed theory.

Definition 2.0. Let $A\left(R^{a} \rightarrow R^{a}\right)$ be the set of affine operators mapping $R^{a}$ to $R^{\alpha}$. Define for all $x, y \in R^{\alpha}$ the mapping $S$ by

$$
S(x) y=A(x) y+B(x) c,
$$

where $A(x) \in L\left(R^{a} \rightarrow R^{a}\right), B(x) \in L\left(R^{p} \rightarrow R^{q}\right)$, and $c \in R^{p}$. We call $S$ an affine operator-valued mapping and write $S \in \tilde{A}\left(R^{q} \rightarrow A\left(R^{q} \rightarrow R^{q}\right)\right)$.

Definition 2.1. Let $S \in \tilde{A}\left(R^{a} \rightarrow A\left(R^{a} \rightarrow R^{a}\right)\right)$ be given by (2.0). We say $S$ is irreducibly diagonally dominant of positive type or I.D.D.P.T., if the matrix $A(x)$ is irreducibly diagonally dominant for all $x \in R^{a}$ and the $q \times(q+p)$ matrix $Q(x)$ defined by

$$
\begin{aligned}
{[Q(x)]_{i j} } & =[A(x)]_{i j}, \quad i, j \leqq q, \\
& =[B(x)]_{i j}, \quad i \leqq q, q+1 \leqq j \leqq q+p,
\end{aligned}
$$

satisfies $[Q(x)]_{i i}<0,[Q(x)]_{i i} \geqq 0, j \neq i, i=1, \cdots, q, j=1, \cdots, q+p$ for all $x \in R^{\alpha}$, and

$$
\left|[Q(x)]_{i i}\right| \geqq \sum_{i=1 ; i \neq i}^{p+a}[Q(x)]_{i i}, \quad i=1, \cdots, q,
$$

for all $x \in R^{a}$.

The next theorem is a generalization of results of Bers [1] and McAllister [6].

THEOREM 2.2. Let $S \in \widetilde{A}\left(R^{a} \rightarrow A\left(R^{a} \rightarrow R^{a}\right)\right)$ be given by (2.0). Assume that $S$ is I.D.D.P.T. and that there exists $v \in R^{a}$ such that for all $x \in R^{a}$

$$
S(x) v \geqq e,
$$

where $e \in R^{a}$ is the vector with one in each component. Then, for all $x \in R^{a},[A(x)]^{-1}$ exists and has nonpositive elements; that is

$$
[A(x)]^{-1} \leqq 0 .
$$

Moreover, the following inequalities hold uniformly for $x \in R^{a}$ :

$$
\left\|[A(x)]^{-1} B(x)\right\|_{\infty} \leqq 1,
$$

and

$$
\left\|[A(x)]^{-1}\right\|_{\infty} \leqq\|v\|_{\infty}+\|c\|_{\infty},
$$

where \|\|$_{\infty}$ is the $l^{\circ}$ operator norm.

Proof. Since the operator $S$ is I.D.D.P.T., the matrix $A(x)$ is irreducibly diagonally dominant for each fixed $x \in R^{a}$. From Collatz [3, p. 45], it follows that $A(x)$ is nonsingular and $[A(x)]^{-1} \leqq 0$. Let $x \in R^{a}$ be fixed but otherwise arbitrary and set

$$
A(x)=D(x)+M(x)
$$


where $D(x)$ is the diagonal part of $A(x)$. If we define

$$
A_{\epsilon}(x)=D(x)-\epsilon I+M(x), \quad \epsilon>0,
$$

then we have

$$
\left\|\left[A_{\epsilon}(x)\right]^{-1} B(x)\right\|_{\infty}=\left\|\left[I+(D(x)-\epsilon I)^{-1} M(x)\right]^{-1}(D(x)-\epsilon I)^{-1} B(x)\right\|_{\infty} .
$$

The diagonal dominance of $S$ (i.e. (2.2)) implies that

$$
\left\|(D(x)-\epsilon I)^{-1} M(x)\right\|_{\infty}<1, \quad \epsilon>0,
$$

so that by Banach's lemma

$$
\left\|\left[A_{\epsilon}(x)\right]^{-1} B(x)\right\|_{\infty} \leqq \frac{\left\|(D(x)-\epsilon I)^{-1} B(x)\right\|_{\infty}}{1-\left\|(D(x)-\epsilon I)^{-1} M(x)\right\|_{\infty}}, \quad \epsilon>0 .
$$

Now,

$$
\left\|(D(x)-\epsilon)^{-1} B(x)\right\|_{\infty}=\max _{1 \leqq i \leqq \alpha} \frac{\sum_{i-1}^{P}[B(x)]_{i i}}{\left|[A(x)]_{i i}-\epsilon\right|} \leqq 1-\max _{1 \leqq i \leqq Q} \frac{\sum_{i-1 ; i \neq i}^{a}[A(x)]_{i i}}{\left|[A(x)]_{i i}-\epsilon\right|},
$$

where the last ine 1 uality follows from the diagonal dominance of $S$. Since

$$
\left\|(D(x)-\epsilon)^{-1} M(x)\right\|_{\infty}=\max _{1 \leqq i \leqq q} \frac{\sum_{i=1 ; j \neq i}^{a}[A(x)]_{i j}}{\left|[A(x)]_{i i}-\epsilon\right|},
$$

we have from (2.8)

$$
\left\|\left[A_{\mathrm{e}}(x)\right]^{-1} B(x)\right\|_{\infty}<1, \quad \epsilon>0 .
$$

Letting $\epsilon \rightarrow 0$ we obtain (2.5) uniformly for all $x \in R^{q}$.

For $y \in R^{\alpha}$, we have the identity

$$
y=-[A(x)]^{-1} B(x) c+[A(x)]^{-1}[S(x) y] .
$$

Let $v \in R^{a}$ satisfy (2.3). Since $[A(x)]^{-1} \leqq 0$, it follows that

$$
\left\|v+[A(x)]^{-1} B(x) c\right\|_{\infty}=\left\|[A(x)]^{-1} S(x) v\right\|_{\infty} \geqq\left\|[A(x)]^{-1} e\right\|_{\infty} .
$$

Then, (2.6) follows from (2.5) and the observation that

$$
\left\|[A(x)]^{-1}\right\|_{\infty} \equiv\left\|[A(x)]^{-1} e\right\|_{\infty} .
$$

THEOREM 2.3. Let $S$ satisfy the hypothesis of Theorem 2.2, and assume that $A(x)$ and $B(x)$ are continuous functions of $x \in R^{\alpha}$. Let $F: R^{\alpha} \rightarrow R^{\alpha}$ be continuous on $R^{\alpha}$ and satisfy for all $x \in R^{a},\|F(x)\|_{\infty} \leqq M<\infty$. Then, there exists a solution to the equation

$$
S(x) x=F(x)
$$

in the sphere

$$
\mathcal{S}=\left\{x \in R^{\alpha} \mid\|x\|_{\infty} \leqq\|c\|_{\infty}+\left(\|v\|_{\infty}+\|c\|_{\infty}\right) M\right\} .
$$

Proof. The Eq. (2.10) is equivalent to the fixed point problem: find $x \in R^{a}$ so that

$$
x=-[A(x)]^{-1} B(x) c+[A(x)]^{-1} F(x) \equiv \hat{F}(x)
$$


From Theorem 2.2, it is clear $\hat{F}(\delta) \subset \delta$. Since $\hat{F}$ is continuous on $\delta$, the conclusion follows by the Brouwer fixed point theorem.

3. The General Discrete Problem. We consider in this section the discrete problem obtained from an arbitrary discretization of the boundary-value problem of Section 1 for operators $L$ that satisfy the following definition:

Definition 3.0. We say $L$ of (1.1) is uniformly elliptic if there exists $c_{1} \geqq c_{0}>0$ such that

$$
\begin{aligned}
c_{1}\left(\eta^{2}+\zeta^{2}\right) & \geqq A(s, t, r, p, q) \eta^{2}+2 B(s, t, r, p, q) \eta \zeta+C(s, t, r, p, q) \zeta^{2} \\
& \geqq c_{0}\left(\eta^{2}+\zeta^{2}\right),
\end{aligned}
$$

for all $(s, t, r, p, q) \in \bar{\Omega} \times R^{3}$ and $\eta, \zeta$ real.

We assume that a square mesh of a side $h$ is placed on $\bar{\Omega}$ and denote by $\Omega_{h}$, the intersections of all grid lines interior to $\Omega$, and number these $P_{1}, \cdots, P_{N}$. Set $\partial \Omega_{h}$ to be the set of all intersections of grid lines with $\partial \Omega$, plus (possibly) any other finite set of points on $\partial \Omega$, and number these $P_{N+1}, \cdots, P_{M}$. Finally, put $\bar{\Omega}_{h}=\Omega_{h} \cup \partial \Omega_{h}$.

We approximate the various derivatives $u_{s}, u_{t}, u_{s s}, u_{s t}, u_{t}$ at each $P_{i} \in \Omega_{b}$ by general linear combinations of $u\left(P_{i}\right) \equiv u_{i}, P_{i} \in \bar{\Omega}_{h}$, that is

$$
u_{s}\left(P_{i}\right) \doteq \sum_{i=1}^{M} a_{i j}^{1} u_{i}, \quad u_{t}\left(P_{i}\right) \doteq \sum_{i=1}^{M} a_{i i}^{2} u_{i},
$$

and

$$
u_{s s}\left(P_{i}\right) \doteq \sum_{i=1}^{M} b_{i j}^{1} u_{i}, \quad u_{s t}\left(P_{i}\right) \doteq \sum_{i=1}^{M} b_{i j}^{2} u_{i}, \quad u_{t t}\left(P_{i}\right) \doteq \sum_{j=1}^{M} b_{i j}^{3} u_{i} .
$$

The coefficients of the linear combinations will, in general, depend on $h$.

Using the approximations (3.1) and (3.2), we replace the boundary-value problem of Section 1 by the system of equations

$$
A_{i} \sum_{i=1}^{M} b_{i j}^{1} u_{i}+2 B_{i} \sum_{i=1}^{M} b_{i j}^{2} u_{i}+C_{i} \sum_{i=1}^{M} b_{i j}^{3} u_{i}=g_{i}, \quad i=1, \cdots, N,
$$

where $A_{i}=A\left(P_{i}, u_{i}, \sum_{i=1}^{M} a_{i j}^{1} u_{i}, \sum_{i=1}^{M} a_{i j}^{2} u_{i}\right)$ and $B_{i}, C_{i}$, and $g_{i}$ are similarly defined.

Define the block matrices

$$
H=\left(H_{1}^{T}, \cdots, H_{N}^{T}\right)^{T}, \quad K=\left(K_{1}^{T}, \cdots, K_{N}^{T}\right)^{T},
$$

where the superscript stands for transpose. Here,

$$
H_{i}=\left[\begin{array}{c}
0, \cdots, 0,1,0, \cdots, 0 \\
a_{i, 1}^{1}, \cdots, \ldots, a_{i, N}^{1} \\
a_{i, 1}^{2}, \cdots \cdots, ., a_{i, N}^{2}
\end{array}\right], \quad K_{i}=\left[\begin{array}{c}
0, \cdots \cdots, 0,0 \\
a_{i, N+1}^{1}, \cdots, a_{i, M}^{1} \\
a_{i, N+1}^{2}, \cdots, a_{i, M}^{2}
\end{array}\right]
$$

and the one in $H_{i}$ appears in the $i$ th position. Denote by $w$ the vector of boundary values with components $w_{i}=u_{i}, j=N+1, \cdots, M$, and define the affine operators $T_{i}: R^{N} \rightarrow R^{3}, i=1, \cdots, N$, and $T: R^{N} \rightarrow R^{3 N}$ by

$$
T_{i} x=H_{i} x+K_{i} w, \quad i=1, \cdots, N, \quad T x=H x+K w .
$$

Let $\widetilde{G}: R^{N} \rightarrow R^{N}$ be given by $[\widetilde{G}(x)]_{i}=g\left(P_{i}, T_{i} x\right), i=1, \cdots, N$. Denote by 
$L_{h}: R^{N} \rightarrow L\left(R^{N} \rightarrow R^{N}\right)$, and $F_{h}: R^{N} \rightarrow L\left(R^{M-N}, R^{N}\right)$, respectively, the matrix-valued mappings defined by

$$
\left[L_{h}(x)\right]_{i j}=A\left(P_{i}, T_{i} x\right) b_{i j}^{1}+2 B\left(P_{i}, T_{i} x\right) b_{i j}^{2}+C\left(P_{i}, T_{i} x\right) b_{i j}^{3},
$$

$i, j=1, \cdots, N$, and

$$
\left[F_{h}(x)\right]_{i j}=A\left(P_{i}, T_{i} x\right) b_{i, i+N}^{1}+2 B\left(P_{i}, T_{i} x\right) b_{i, i+N}^{2}+C\left(P_{i}, T_{i} x\right) b_{i, j+N}^{3},
$$

$i=1, \cdots, N, j=1, \cdots, M-N$. If we set $x \in R^{N}$ to be the vector with components $u_{i}, i=1, \cdots, N$, then the system of equations (3.3) is given by

$$
L_{h}(x) x+F_{h}(x) w=\tilde{G}(x) .
$$

Let us define $\Upsilon_{h} \in \tilde{A}\left(R^{N} \rightarrow A\left(R^{N} \rightarrow R^{N}\right)\right)$ by

$$
\Upsilon_{h}(y) z=L_{h}(y) z+F_{h}(y) w .
$$

Then, (3.3) is equivalent to

$$
\Upsilon_{h}(x) x=\tilde{G}(x) .
$$

We now have the problem in a form where the results of the previous section can be applied.

Definition 3.1. Let $\delta$ stand for some first or second derivative operator, $\delta_{h}(P): C(\bar{\Omega}) \rightarrow R^{1}$, for all $P \in \Omega$. Then $\delta_{h}$ is consistent with $\delta$ if any $u \in C^{2}(\bar{\Omega})$

$$
\lim _{h \rightarrow 0}\left|\delta_{h}(P) u-(\delta u)(P)\right|=0
$$

uniformly over $\Omega$.

LEMMA 3.2. Let

$$
L_{1} u=L u+d\left(s, t, u, u_{t}, u_{t}\right) u_{s}+e\left(s, t, u, u_{s}, u_{t}\right) u_{t}-f\left(s, t, u, u_{s}, u_{t}\right) u,
$$

where $L$ is the uniformly elliptic operator given by (1.1) and

$$
\begin{aligned}
|d(s, t, r, p, q)|,|e(s, t, r, p, q)| & \leqq c \leqq \infty, \\
f(s, t, r, p, q) & \geqq 0,
\end{aligned}
$$

uniformly on $\bar{\Omega} \times R^{3}$. Assume we discretize $L$, with consistent approximations to the first and second derivatives. Then, there exists $v_{h} \in R^{N}$ such that for all $h$ sufficiently small

$$
\hat{\Upsilon}_{h}(x) v_{h} \geqq e
$$

for all $x \in R^{N}$, and $\left\|v_{h}\right\|_{\infty}$ is uniformly bounded in $h$. Here, of course, $\hat{\Upsilon}_{h}$ is the operator corresponding to the discretization of $L_{1}$.

Proof. Suppose, without loss of generality, that $\bar{\Omega}$ is included in the strip $0 \leqq s \leqq k^{*}$. Let $k(s, t)=e^{\beta s}-e^{\alpha}$ with $\beta=2 c / c_{0}$ and $\alpha>\beta k^{*}$. Here, $c_{0}$ is the smaller constant of uniform ellipticity. Observe that, $1-e^{\alpha} \leqq k(s, t) \leqq 0$. Define $\bar{v}_{h} \in R^{N}$ as the vector with components $\left(v_{h}\right)_{i}=k\left(P_{i}\right), i=1, \cdots, N$, and note that $\left\|\bar{v}_{h}\right\|_{\infty} \leqq e^{\alpha}-1$, uniformly in $h$. Now, choose $x \in R^{N}$ arbitrary and calculate

$$
\begin{aligned}
{\left[\hat{\Upsilon}_{h}(x) \bar{v}_{h}\right]_{m} \geqq } & {\left[\beta^{2} A\left(P_{m}, T_{m} x\right)+\beta d\left(P_{m}, T_{m} x\right)\right] e^{\beta_{s i}}+f\left(P_{m}, T_{m} x\right)\left[e^{\alpha}-e^{\beta_{s i}}\right] } \\
& -\mid\left[\Upsilon_{h}(x) v_{h}\right]_{m}-\left[\beta^{2} A\left(P_{m}, T_{m} x\right)+\beta d\left(P_{m}, T_{m} x\right)\right] e^{\beta_{s i}} \\
& -f\left(P_{m}, T_{m} x\right)\left[e^{\alpha}-e^{\beta_{s i}}\right] \mid .
\end{aligned}
$$


Here, $P_{m} \equiv\left(s_{i}, t_{j}\right)$. Since the approximations to the derivatives are consistent, we have for all $h$ sufficiently small, and $P \in \Omega_{h}$, that the last term in the above expression does not exceed $c^{2} / c_{0}$. Then, since the sum of the first two terms is not smaller than $2 c^{2} / c_{0}$, we may conclude

$$
\left[\hat{\Upsilon}_{h}(x) \bar{v}_{h}\right]_{m} \geqq c^{2} / c_{0}
$$

for all $P_{m} \in \Omega_{h}$, and $h$ sufficiently small. Thus, the vector $v_{h}=c_{0} \bar{v}_{h} / c^{2}$ satisfies (3.7) for all $x \in R^{N}$ and $\left\|v_{h}\right\|_{\infty} \leqq c_{0}\left(e^{\alpha}-1\right) / c^{2}$.

The proof of the following existence theorem is immediate from Theorem 2.3 and Lemma 3.2.

THEOREM 3.3. Consider the boundary-value problem of Section 1 for uniformly elliptic $L_{1}$ instead of $L$. Assume we discretize $L_{1}$ with consistent approximations to the first and second derivatives. Assume further that the resulting operator $\hat{\Upsilon}_{h}$ is I.D.D.P.T. for all $h$ sufficiently small, and in addition $L_{h}, F_{h}$ and $\widetilde{G}$ are continuous functions of $x \in R^{N}$ for all $h$ sufficiently small. Let $g: \bar{\Omega} \times R^{3} \rightarrow R^{1}$ satisfy $|g(s, t, r, p, q)| \leqq$ $M<\infty$, uniformly on $\bar{\Omega} \times R^{3}$. Then, for $h$ sufficiently small the discrete analogue of the boundary-value problem has a solution in the sphere

$$
\mathcal{S}=\left\{x \in R^{N} \mid\|x\|_{\infty} \leqq\|w\|_{\infty}+\left(2 c_{0}\left[e^{\alpha}-1\right] / c^{2}\right) M\right\},
$$

where $\alpha$ and $c$ are defined in Lemma 3.2.

We have proved a result for a slightly more general operator than (3.6), because it will be useful in the next section.

4. An I.D.D.P.T. Operator. In this section, it will be convenient to have a doubly subscripted notation for the gridpoints as well as the singly subscripted notation of the previous section. We effect this by the following procedure: number the rows and columns of $\bar{\Omega}_{h}$ from left to right, bottom to top, and denote by $P_{i i}$ the element in the $i$ th row and $j$ th column. Then, any gridpoint may be represented in the equivalent notation $P_{k}$ or $P_{i}$.

For a linear uniformly elliptic operator $L$ of the form

$$
L u=A(s, t) u_{s s}+2 B(s, t) u_{s t}+c(s, t) u_{t t},
$$

Bramble and Hubbard [2] consider the discretization

(a) $u_{s s}\left(P_{i j}\right) \doteq \frac{u_{i+1, j}-2 u_{i j}+u_{i-1, i}}{h^{2}} \equiv u_{i s}\left(P_{i j}\right)$,

(b) $u_{t i}\left(P_{i j}\right) \doteq \frac{u_{i, i+1}-2 u_{i j}+u_{i, j-1}}{h^{2}} \equiv u_{i t}\left(P_{i j}\right)$,

(c) $u_{s t}\left(P_{i j}\right) \doteq \frac{\alpha^{2}+\beta^{2}}{2 \alpha \beta} \operatorname{sgn}\left(B\left(P_{i j}\right)\right)\left[u_{i z}\left(P_{i j}\right)-\frac{\beta^{2}}{\alpha^{2}+\beta^{2}} u_{i s}\left(P_{i j}\right)\right.$

$$
\left.-\frac{\alpha^{2}}{\alpha^{2}+\beta^{2}} u_{i t}\left(P_{i j}\right)\right] \text {, }
$$

where $u_{i j}=u\left(P_{i j}\right)$ and

$$
u_{\bar{z} \Sigma}\left(P_{i j}\right)=\frac{u_{i \pm \beta, j+\alpha}-2 u_{i i}+u_{i \mp \beta, j-\alpha}}{h^{2}\left(\alpha^{2}+\beta^{2}\right)} .
$$


Here, $\alpha$ and $\beta$ are relatively prime integers that may be functions of the point $P_{i j}$ and in (4.2) we use the points $P_{i+\beta, j+\alpha}, P_{i-\beta, j-\alpha}$ if $B\left(P_{i j}\right) \geqq 0$ and the points $P_{i-\beta, i+\alpha}$, $P_{i+\beta, j-\alpha}$ if $B\left(P_{i j}\right)<0$.

Bramble and Hubbard [2] show that the discretization (4.1) always leads to a $\Upsilon_{h}$ which is I.D.D.P.T. Their main result that allows this conclusion can be summarized as the following:

THEOREM 4.0. Suppose $L$ of (4.0) is uniformly elliptic and has continuous coefficients $A(s, t), B(s, t)$ and $C(s, t)$ for $(s, t) \in \bar{\Omega}$. Then, there exists constants $k_{0}^{\prime}$ and $\eta$ with $k_{0}^{\prime}>0$, and $1 \leqq \eta<\infty$ and a mapping $\gamma: \Omega \rightarrow(-\infty, \infty)$ such that for all $(s, t) \in \Omega$

$$
\begin{aligned}
& A^{\prime}(s, t)=A(s, t)-\frac{B(s, t)}{\gamma(s, t)} \geqq k_{0}^{\prime}, \\
& C^{\prime}(s, t)=C(s, t)-\gamma(s, t) B(s, t) \geqq k_{0}^{\prime}, \\
& B^{\prime}(s, t)=\frac{\gamma^{2}(s, t)+1}{2 \gamma(s, t)} B(s, t) \geqq 0 .
\end{aligned}
$$

Moreover, $\gamma$ can be chosen so that $|\gamma(s, t)|=\alpha(s, t) / \beta(s, t)$, where $\alpha(s, t)$ and $\beta(s, t)$ are relatively prime integers satisfying

$$
\alpha(s, t) \geqq 1, \quad \beta(s, t) \leqq \eta
$$

for all $(s, t) \in \Omega$.

It is easy to see why a result like Theorem 4.0 is important, because, if we substitute the discretization (4.1) into (4.0), then the coefficients of the discretized operator are just $A^{\prime}, B^{\prime}$ and $C^{\prime}$ of (4.3).

For quasi-linear uniformly elliptic operators, McAllister [6] showed that if the coefficients of $L$ satisfy the inequalities

$$
\begin{array}{r}
\infty>a_{1} \geqq A(s, t, r, p, q), C(s, t, r, p, q) \geqq a_{0}>0, \\
2|B(s, t, r, p, q)|<a_{0},
\end{array}
$$

for all (s,t,r,p,q) $\in \bar{\Omega} \times R^{3}$, then the discretization (4.1) (a) and (b) and

$$
\begin{aligned}
h^{2} u_{s t}\left(P_{i j}\right) & \doteq u_{i+1, i+1}-u_{i+1, i}-u_{i, i+1}+u_{i j}, & & B\left(P_{i j}\right) \geqq 0, \\
& \doteq u_{i, i+1}-u_{i j}-u_{i+1, i-1}+u_{i-1, i}, & & B\left(P_{i j}\right)<0,
\end{aligned}
$$

gives rise to a $\Upsilon_{h}$ which is I.D.D.P.T. This is somewhat unsatisfactory, because of the very restrictive nature of (4.5). Frank [4] attempted to overcome this restriction by extending the Bramble and Hubbard discretization to all quasi-linear uniformly elliptic operators of the form (1.1). The tool Frank used for proving this, was the claim that there exists a mapping $\gamma: \Omega \rightarrow(-\infty, \infty)$ with all the properties of Theorem 4.0, for $L$ whose coefficients $A, B$ and $C$ depend on $u, u_{\text {, and }} u_{t}$ as well as $s$ and $t$. Unfortunately, this is untrue. Consider the following uniformly elliptic operator:

$$
L u=\left(\frac{1}{2}+\sin ^{2} u\right) u_{s s}+\frac{4}{3} u_{s t}+\left(\frac{1}{2}+\cos ^{2} u\right) u_{t t}
$$

At $(\pi / 2)$ we have

$$
A\left(\frac{\pi}{2}\right)=\frac{3}{2}>B\left(\frac{\pi}{2}\right)=\frac{2}{3}>C\left(\frac{\pi}{2}\right)=\frac{1}{2}
$$


while at 0 we have

$$
A(0)=\frac{1}{2}<B(0)=\frac{2}{3}<C(0)=\frac{3}{2} .
$$

Thus, to satisfy an analogue of Theorem $4.0, \gamma$ would have to be both strictly less than one and strictly greater than one, an absurdity. We will show that an analogue of Theorem 4.0 holds if and only if the coefficients of $L$ satisfy the following condition:

$$
\begin{aligned}
W(s, t) & \equiv \inf _{(r, p, q) \in R^{s}} \frac{C(s, t, r, p, q)}{|B(s, t, r, p, q)|} \\
& >\sup _{(r, p, q) \in R^{s}} \frac{|B(s, t, r, p, q)|}{A(s, t, r, p, q)} \equiv w(s, t)
\end{aligned}
$$

for all $(s, t) \in \bar{\Omega}$.

If $A, B$, and $C$ depend only on $(s, t) \in \bar{\Omega}$, then the condition follows as an immediate consequence of uniform ellipticity. However, for the quasi-linear case, this is not true, as (4.7) shows. What is always true, of course, is that for fixed $(r, p, q) \in R^{3}$

$$
\frac{C(s, t, r, p, q)}{|B(s, t, r, p, q)|}>\frac{|B(s, t, r, p, q)|}{A(s, t, r, p, q)}
$$

Thus, what (4.8) demands is that the relative magnitude of the left- and right-hand side of (4.9) do not vary "greatly" for fixed $(s, t) \in \bar{\Omega}$. In the special case that $B$ is "small" (i.e. satisfies (4.5)) it is clear that (4.8) holds.

An analogue of Theorem 4.0 for quasi-linear $L$ will now be proven.

THEOREM 4.1. Suppose $L$ is uniformly elliptic. Then there exist $k_{0}^{\prime}>0$ and $1 \leqq$ $\eta<\infty$, independent of $(s, t) \in \bar{\Omega}$, and a mapping $\gamma: \bar{\Omega} \rightarrow(0, \infty)$ such that

$$
\begin{aligned}
& A^{\prime}(s, t, r, p, q)=A(s, t, r, p, q)-\frac{|B(s, t, r, p, q)|}{\gamma(s, t)} \geqq k_{0}^{\prime}, \\
& C^{\prime}(s, t, r, p, q)=C(s, t, r, p, q)-\gamma(s, t)|B(s, t, r, p, q)| \geqq k_{0}^{\prime}, \\
& B^{\prime}(s, t, r, p, q)=\frac{\gamma^{2}(s, t)+1}{2 \gamma(s, t)}|B(s, t, r, p, q)| \geqq 0,
\end{aligned}
$$

for all $(s, t, r, p, q) \in \bar{\Omega} \times R^{3}$, if and only if, (4.8) holds for all $(s, t) \in \bar{\Omega}$. Moreover, $\gamma$ can be chosen as $\gamma(s, t)=\alpha(s, t) / \beta(s, t)$, where $\alpha(s, t)$ and $\beta(s, t)$ are relatively prime integers satisfying $\alpha(s, t) \geqq 1, \beta(s, t) \leqq \eta$ for all $(s, t) \in \bar{\Omega}$, and $\gamma(s, t) \in(w(s, t)$, $W(s, t))$.

Proof. Without loss of generality, assume $B(s, t, r, p, q) \not \equiv 0$ for all $(s, t) \in \bar{\Omega}$. Since (4.8) holds, and $\bar{\Omega}$ is compact, we may choose

$$
0<\epsilon^{*}<\min \left\{\inf _{(s, t) \in \bar{\Omega}} \frac{W(s, t)-w(s, t)}{2}, c_{0}\right\},
$$

where $c_{0}>0$ is the smaller constant of uniform ellipticity. If at a point $\left(s_{0}, t_{0}\right) \in \bar{\Omega}$, $B\left(s_{0}, t_{0}, r, p, q\right) \neq \equiv$, then choose

$$
\gamma\left(s_{0}, t_{0}\right) \in\left[w\left(s_{0}, t_{0}\right)+\epsilon^{*}, W\left(s_{0}, t_{0}\right)-\epsilon^{*}\right] .
$$

Then, 


$$
\begin{aligned}
A\left(s_{0}, t_{0}, r, p, q\right)-\frac{\left|B\left(s_{0}, t_{0}, r, p, q\right)\right|}{\gamma\left(s_{0}, t_{0}\right)} & =\frac{A\left(s_{0}, t_{0}, r, p, q\right) \gamma\left(s_{0}, t_{0}\right)-\left|B\left(s_{0}, t_{0}, r, p, q\right)\right|}{\gamma\left(s_{0}, t_{0}\right)} \\
& \geqq \frac{A\left(s_{0}, t_{0}, r, p, q\right) \epsilon^{*}}{\gamma\left(s_{0}, t_{0}\right)} \\
& \geqq \frac{c_{0} \epsilon^{*}}{W\left(s_{0}, t_{0}\right)-\epsilon^{*}} .
\end{aligned}
$$

The first inequality follows from (4.11), while the second follows from (4.11), and the uniform ellipticity of $L$. Assume next that

$$
\left|B\left(s_{0}, t_{0}, r, p, q\right)\right|<\frac{c_{0}}{2\left(W\left(s_{0}, t_{0}\right)-\epsilon^{*}\right)} .
$$

Then, it follows from (4.11) and uniform ellipticity that

$$
C\left(s_{0}, t_{0}, r, p, q\right)-\gamma\left(s_{0}, t_{0}\right)\left|B\left(s_{0}, t_{0}, r, p, q\right)\right| \geqq c_{0} / 2 .
$$

If

$$
B\left(s_{0}, t_{0}, r, p, q\right) \geqq \frac{c_{0}}{2\left(W\left(s_{0}, t_{0}\right)-\epsilon^{*}\right)},
$$

then, by (4.11) and uniform ellipticity

$$
C\left(s_{0}, t_{0}, r, p, q\right)-\gamma\left(s_{0}, t_{0}\right)\left|B\left(s_{0}, t_{0}, r, p, q\right)\right| \geqq \frac{c_{0} \epsilon^{*}}{2\left(W\left(s_{0}, t_{0}\right)-\epsilon^{*}\right)} \cdot
$$

Now, it is clear there exists a $\gamma: \bar{\Omega} \rightarrow(0, \infty)$ satisfying (4.10) for all $(s, t, r, p, q) \in$ $\bar{\Omega} \times R^{3}$, where

$$
k_{0}^{\prime}=\frac{c_{0}}{2} \min \left\{1, \inf _{(s, t) \in \bar{\Omega}} \frac{\epsilon^{*}}{\left(W(s, t)-\epsilon^{*}\right)}\right\}>0 .
$$

Set

$$
z=2 \min \left\{\inf _{(s, t) \in \bar{\Omega}} \frac{W(s, t)-w(s, t)}{2}, c_{0}\right\}-2 \epsilon^{*} .
$$

By definition of $\epsilon^{*}, z>0$. If $z \geqq 1$, then we may choose $\eta \equiv 1$, since every interval $\left[w\left(s_{0}, t_{0}\right)+\epsilon^{*}, W\left(s_{0}, t_{0}\right)-\epsilon^{*}\right],\left(s_{0}, t_{0}\right) \in \bar{\Omega}$, will contain an integer. If $z<1$, then choose an integer $k$ such that $10^{-k}<z$; then, there exists an integer $\tilde{\alpha}\left(s_{0}, t_{0}\right)$ such that $\tilde{\alpha}\left(s_{0}, t_{0}\right) 10^{-k} \in\left[w\left(s_{0}, t_{0}\right)+\epsilon^{*}, W\left(s_{0}, t_{0}\right)-\epsilon^{*}\right]$. Thus, if we choose

$$
\eta=\left[\frac{1}{z}+1\right]
$$

where [ ] means greatest integer, the "if" proof of the theorem will follow, since if $\tilde{\alpha}\left(s_{0}, t_{0}\right)$ and $10^{k}$ are not relatively prime, we may divide out the common factors without changing (4.13).

If we assume there exists a $\gamma: \bar{\Omega} \rightarrow(0, \infty)$, such that (4.10) holds for all $(s, t, r, p, q)$ $\in \bar{\Omega} \times R^{3}$, then we have for $B\left(s_{0}, t_{0}, r, p, q\right) \not \equiv 0$ that

$$
\frac{C\left(s_{0}, t_{0}, r, p, q\right)}{\left|B\left(s_{0}, t_{0}, r, p, q\right)\right|}-\tilde{k} \geqq \gamma\left(s_{0}, t_{0}\right) \geqq \frac{\left|B\left(s_{0}, t_{0}, r, p, q\right)\right|}{A\left(s_{0}, t_{0}, r, p, q\right)-k_{0}^{\prime}}
$$


where

$$
\bar{k}=k_{0}^{\prime} / c_{1}
$$

and $c_{1}$ is the larger constant of uniform ellipticity. This follows immediately, since uniform ellipticity implies $|B(s, t, r, p, q)|<c_{1}$ for all $(s, t, r, p, q) \in \bar{\Omega} \times R^{3}$. Now, (4.8) is a direct consequence of (4.14).

The next theorem, whose proof is analogous in the idea to that of Theorem 4.1, gives necessary and sufficient conditions so that $\gamma(s, t)$ may be picked independent of $(s, t) \in \bar{\Omega}$, as well as $(r, p, q) \in R^{3}$.

THEOREM 4.2. Suppose L satisfies the hypothesis of the last theorem. Then, there exist relatively prime integers $\alpha$ and $\beta$ with $\gamma(s, t) \equiv \alpha / \beta$ for all $(s, t) \in \bar{\Omega}$, and $a$ constant $k_{0}^{\prime}>0$, so that (4.10) is satisfied for all $(s, t, r, p, q) \in \bar{\Omega} \times R^{3}$, if and only if,

$$
\bar{W}=\inf _{(1, t) \in \bar{\Omega}} W(s, t)>\sup _{(s, t) \in \bar{\Omega}} w(s, t)=\bar{w} .
$$

We now proceed to show that the discretization (4.1) applied to a uniformly elliptic operator satisfying the conditions of Theorem 4.1 yields an operator $\Upsilon_{h}$ which is I.D.D.P.T. We first need some preliminary definitions.

Definition 4.3. We call a mesh $\Omega_{h}$ connected, if given any two mesh points $P_{i}$ and $P_{i} \in \Omega_{h}$, then there exists a polygonal arc contained in $\bar{\Omega}$ which consists of straight line segments of constant length $h$ starting at $P_{i}$ and ending at $P_{i}$, each of whose endpoints is in $\Omega_{h}$.

Definition 4.4. Suppose $W(s, t)>w(s, t)$ for all $(s, t) \in \bar{\Omega}$. Choose $\alpha(s, t)$ and $\beta(s, t)$ by Theorem 4.1. We call a mesh regular, if given any point $P_{m, n} \in \Omega_{h}$, then the points $P_{m, n \pm 1}, P_{m \pm 1, n}$, as well as the points $P_{m \pm \beta, n+\alpha}$, and $P_{m \pm \beta, i-\alpha}$, are in $\bar{\Omega}_{h}$. Here $\alpha=$ $\alpha\left(P_{m, n}\right)$ and $\beta=\beta\left(P_{m, n}\right)$.

If the mesh does not satisfy Definition 4.4 , then it will be necessary to alter the discretization at the nonregular points. This can be done as in Bramble and Hubbard [2]. For example, suppose $P_{i+1, i} \notin \bar{\Omega}$. Then there exists $\lambda<1$ such that $P_{i+\lambda, i} \in \partial \Omega$ and is on the line joining $P_{i j}$ to $P_{i+1, j}$. We approximate

$$
u_{s s}\left(P_{i j}\right) \doteq \frac{2}{h^{2}}\left[\frac{1}{\lambda+1} u_{i-1, i}-\frac{1}{\lambda} u_{i j}+\frac{1}{\lambda(\lambda+1)} u_{i+\lambda, i}\right] \text {. }
$$

If any of the other points are not in $\bar{\Omega}_{h}$ similar formulas are used. For the first derivatives similar redefinitions would, in general, be needed. Suppose we used the central difference approximation

$$
u_{s}\left(P_{i j}\right) \doteq \frac{u_{i+1, i}-u_{i-1, i}}{2 h} .
$$

Then, if $P_{i+1, i} \notin \bar{\Omega}$ we would define the new approximation

$$
u_{s}\left(P_{i j}\right) \doteq \frac{u_{i+\lambda, j}-u_{i-1, i}}{(\lambda+1) h}
$$

LEMMA 4.5. Let $u \in C^{2}(\bar{\Omega})$. Then the discretization

$$
u_{s s}(s, t) \doteq \frac{2}{h^{2}}\left[\frac{u(s-h, t)}{\lambda+1}-\frac{u(s, t)}{\left.\lambda+\frac{u(s+\lambda h, t)}{\lambda(\lambda+1)}\right]}\right.
$$


is consistent. Here $\lambda \equiv 1$, if $u(s+h, t) \in \bar{\Omega}$; otherwise $\tilde{\lambda}=\lambda h$ is the distance to the boundary along the line $t$ constant through $(s, t) \in \Omega$.

Proof. Since $u \in C^{2}(\bar{\Omega})$, we expand the right-hand side of (4.20) (i.e. $\left(\delta_{h}(s, t) u\right)$ ) by the Taylor series. Then we obtain

$$
\left|u_{s s}(s, t)-\left(\delta_{h}(s, t) u\right)\right|=\left|u_{s s}(s, t)-\frac{u_{s s}\left(s-\theta_{1} h, t\right)+u_{s s}\left(s+\theta_{2} h, t\right) \lambda}{\lambda+1}\right|,
$$

where $0<\theta_{1}, \theta_{2}<1$. Then noting that $u_{s s}$ is uniformly continuous on $\bar{\Omega}$, we have

$$
\lim _{h \rightarrow 0}\left|u_{s s}(s, t)-\left(\delta_{h}(s, t) u\right)\right|=0
$$

uniformly over $(s, t) \in \Omega$.

Similar reasoning shows that the other discretizations (4.1) (b) and (4.1) (c) are consistent (when modified, where necessary, in a manner analogous to (4.20)).

THEOREM 4.6. Suppose $L$ is uniformly elliptic and (4.8) is satisfied for all $(s, t) \in \bar{\Omega}$. Let $\alpha=\alpha\left(P_{i j}\right)$ and $\beta=\beta\left(P_{i j}\right)$ be chosen to satisfy Theorem 4.1. Suppose in addition $\Omega_{h}$ is connected. If we discretize the second derivatives by (4.1) and the first derivatives by any consistent discretization, then $L$ yields $a \Upsilon_{h}$ defined by (3.5), which is I.D.D.P.T.

Proof. For clarity, we prove the theorem only for the case $\Omega_{h}$ regular; the modifications for a nonregular mesh are immediate. Choose $y \in R^{N}$. Then

$$
\begin{aligned}
h^{2}\left[\Upsilon_{h}(y) x\right]_{k}= & -2\left(A_{i j}^{\prime}+B_{i j}^{\prime}+C_{i j}^{\prime}\right) x_{i j}+A_{i j}^{\prime}\left(x_{i+1, j}+x_{i-1, i}\right) \\
& +C_{i j}^{\prime}\left(x_{i, j+1}+x_{i, j-1}\right)+B_{i j}^{\prime}\left(x_{i+\beta, i+\alpha}+x_{i-\beta, j-\alpha}\right), \quad B_{i j} \geqq 0, \\
= & -2\left(A_{i j}^{\prime}+B_{i j}^{\prime}+C_{i j}^{\prime}\right) x_{i j}+A_{i j}^{\prime}\left(x_{i+1, i}+x_{i-1, j}\right) \\
& +C_{i j}^{\prime}\left(x_{i, j+1}+x_{i, j-1}\right)+B_{i j}^{\prime}\left(x_{i+\beta, i-\alpha}+x_{i-\beta, i+\alpha}\right), \quad B_{i j}<0 .
\end{aligned}
$$

Here,

$$
\begin{aligned}
& A_{i j}^{\prime}=A_{i j}-\frac{\beta}{\alpha}\left|B_{i j}\right|, \\
& B_{i j}^{\prime}=\frac{\left|B_{i j}\right|}{\alpha \beta}, \\
& C_{i j}^{\prime}=C_{i j}-\frac{\alpha}{\beta}\left|B_{i j}\right|,
\end{aligned}
$$

where $A_{i j}=A\left(P_{i j}, T_{k} y\right)$, and $P_{k} \equiv P_{i j}$. Theorem 4.1 gives that $A_{i j}^{\prime}, C_{i j}^{\prime} \geqq k_{0}^{\prime}>0$ for all $P_{i j} \in \Omega_{h}$, so that the sign relationship of Definition 2.1 is satisfied. Choose any $P_{i j}$ and $P_{m, n} \in \Omega_{h}$. By assumption, there exists a collection of endpoints satisfying Definition 4.3. Since the coefficients of $x_{i \pm 1, i}$ and $x_{i, i \pm 1}$ are all nonzero, we have from a basic result of graph theory (see Varga [9, p. 20, Theorem 1.6]) that $L_{h}(y)$ is irreducible for all $y \in R^{N}$. That $L_{h}(y)$ is in fact irreducibly diagonally dominant follows from (4.21), since some point $P_{l, m} \in \partial \Omega_{h}$ and the $x_{l, m}$ corresponding to it is in $w$, the vector of boundary values. Thus,

$$
\left|L_{h}(y)\right|_{i i} \geqq \sum_{i=1 ; i \neq i}^{N}\left[L_{h}(y)\right]_{i j}, \quad i=1, \cdots, N,
$$

with strict inequality for some $i$. The inequality (2.2) follows immediately from (4.21). 
Since it is clear from (4.21) that $L_{h}$ and $F_{h}$ are continuous functions of $x \in R^{N}$, the last result and Lemma 4.5 imply that Theorem 3.3 holds for discretization (4.1). However, we can obtain a more interesting result for this particular discretization; that is, we can replace the requirement, $|g(s, t, r, p, q)| \leqq M<\infty$, by the condition of Bers [1].

THEOREM 4.7. Suppose the hypothesis of Theorem 4.6 is satisfied. Moreover, suppose $A, B$, and $C \in C\left(\bar{\Omega} \times R^{3}\right)$, and $g \in C^{1}\left(\bar{\Omega} \times R^{3}\right)$ satisfy

$$
\begin{aligned}
\left|g_{p}(s, t, r, p, q)\right|,\left|g_{q}(s, t, r, p, q)\right| & \leqq c<\infty, \\
g_{r}(s, t, r, p, q) & \geqq 0,
\end{aligned}
$$

uniformly in $\bar{\Omega} \times R^{3}$. Assume we discretize the first derivatives by central differences. Then for $h$ sufficiently small (3.6) has a solution.

Proof. We again prove the theorem for regular meshes; the nonregular case requires only minor changes. We show this problem is equivalent to a problem which satisfies Theorem 3.3. By the Mean Value Theorem

$$
\Upsilon_{h}(x) x=G(0)+\int_{0}^{1} G^{\prime}(\theta T x) T x d \theta
$$

where $G: R^{3 N} \rightarrow R^{N}$ is defined by $[G(x)]_{i}=g\left(P_{i}, x_{3 i-2}, x_{3 i-1}, x_{3 i}\right)$. Define the operator $\Upsilon_{h, 1} \in \widetilde{A}\left(R^{N} \rightarrow A\left(R^{N}, R^{N}\right)\right)$ by

$$
\Upsilon_{h, 1}(y) x=L_{h, 1}(y) x+F_{h, 1}(y) w,
$$

where

$$
\begin{aligned}
& L_{h, 1}(x)=L_{h}(x)-\int_{0}^{1} G^{\prime}(\theta T x) H d \theta \\
& F_{h, 1}(x)=F_{h}(x)-\int_{0}^{1} G^{\prime}(\theta T x) K d \theta
\end{aligned}
$$

and $H, K$ are the matrices in (3.4). Then to prove (3.6) has a solution we need only show, by (4.24), that the equation

$$
\Upsilon_{h, 1}(x) x=G(0)
$$

has a solution. However, with $P_{k} \equiv P_{i i}$, we have

$$
\begin{aligned}
{\left[\Upsilon_{h, 1}(y) x\right]_{k}=} & {\left[\Upsilon_{h}(y) x\right]_{k}-g_{k, r} x_{i j}-\frac{1}{2 h} g_{k, p}\left[x_{i+1, i}-x_{i-1, i}\right] } \\
& -\frac{1}{2 h} g_{k, q}\left[x_{i, i+1}-x_{i, i-1}\right]
\end{aligned}
$$

where $g_{k, r}=\int_{0}^{1} g_{r}\left(P_{k}, \theta T_{k} y\right) d \theta$; and $g_{k, p}, g_{k, q}$ are similarly defined. Hence, $\left|g_{k, p}\right|$, $\left|g_{k, a}\right| \leqq c$ and $g_{k, r} \geqq 0$. Since $A_{i j}^{\prime}, C_{i j}^{\prime} \geqq k_{0}^{\prime}>0$, we have from (4.21) and the result that $\Upsilon_{h}$ is I.D.D.P.T. for $h$ sufficiently small, that for $h$ sufficiently small and $h<2 k_{0}^{\prime} / c, \Upsilon_{h, 1}$ is I.D.D.P.T. Then the existence of the solution follows from Theorem 3.3, since $\Upsilon_{h, 1}$ is just the discretization of the operator 


$$
\begin{aligned}
L_{1} u= & L u-\int_{0}^{1} g_{p}\left(s, t, \theta u, \theta u_{s}, \theta u_{t}\right) d \theta u_{s} \\
& -\int_{0}^{1} g_{a}\left(s, t, \theta u, \theta u_{s}, \theta u_{t}\right) d \theta u_{t}-\int_{0}^{1} g_{r}\left(s, t, \theta u, \theta u_{s}, \theta u_{t}\right) d \theta u .
\end{aligned}
$$

Before we close we wish to point out that the results in this section as well as the last depended heavily on $L$ being uniformly elliptic. However, this assumption can be changed to $L$ elliptic without disturbing the results of this paper, if we are willing to make the following stringent assumptions:

(a) $\Omega$ is a convex Jordan region.

(b) $\psi$ satisfies the three-point condition on $\partial \Omega$.

(c) If $\delta_{h}$ stands for the approximation to any second derivative and $l$ is a linear function, then $\delta_{h}(P) l \equiv 0$ for all $P \in \Omega_{h}$, i.e. $\delta_{h}$ is exact on linear functions.

(d) $g(s, t, r, p, q) \equiv 0$.

A proof of this result can be found in Stepleman [8].

Department of Applied Mathematics and Computer Science

University of Virginia

Charlottesville, Virginia 22901

1. L. BERS, "On mildly nonlinear partial differential equations of elliptic type," J. Res. Nat. Bur. Standards Sect. B, v. 5, 1963, pp. 229-236.

2. J. BRAMBle \& B. Hubbard, "A theorem on error estimation for finite difference analogues of the Dirichlet problem for elliptic equations," Contributions to Differential Equations, v. 2, 1963, pp. 319-340. MR 27 \#2114.

3. L. Collatz, Numerische Behandlung von Differentialgleichungen, Die Grundlehren der math. Wissenschaften, Band 60, Springer-Verlag, Berlin, 1951; English transl., SpringerVerlag, Berlin, 1960. MR 13, 285; MR 22 \#322.

4. T. Frank, Error Bounds on Numerical Solutions of Dirichlet Problems for QuasiLinear Elliptic Equations, Thesis, University of Texas, Austin, Tex., 1967.

5. G. MCALlISTER, "Some nonlinear elliptic partial differential equations and difference equations," J. Soc. Indust. Appl. Math., v. 12, 1964, pp. 772-777. MR 31 \#195.

6. G. MCAllisteR, "Quasilinear uniformly elliptic partial differential equations and difference equations," Siam J. Numer. Anal., v. 3, 1966, no. 1, pp. 13-33. MR 34 \#2213.

7. T. MotZKIN \& W. WASOW, "On the approximation of linear elliptic differential equations by difference equations with positive coefficients," J. Mathematical Phys., v. 31, 1953, pp. 253-259. MR 14, 693.

8. R. StePleman, Finite Dimensional Analogues of Variational and Quasi-Linear Elliptic Dirichlet Problems, Thesis, Technical Report \#69-88, Computer Science Center, University of Maryland, College Park, Md., 1969.

9. R. S. VARGA, Matrix Iterative Analysis, Prentice-Hall, Englewood Cliffs, N. J., 1962. MR $28 \# 1725$. 